\title{
Euler's Polyhedron Formula
}

\author{
Jesse Alama \\ Department of Philosophy \\ Stanford University \\ USA
}

Summary. Euler's polyhedron theorem states for a polyhedron $p$, that

$$
V-E+F=2,
$$

where $V, E$, and $F$ are, respectively, the number of vertices, edges, and faces of $p$. The formula was first stated in print by Euler in 1758 [11]. The proof given here is based on Poincaré's linear algebraic proof, stated in [17] (with a corrected proof in [18]), as adapted by Imre Lakatos in the latter's Proofs and Refutations [15].

As is well known, Euler's formula is not true for all polyhedra. The condition on polyhedra considered here is that of being a homology sphere, which says that the cycles (chains whose boundary is zero) are exactly the bounding chains (chains that are the boundary of a chain of one higher dimension).

The present proof actually goes beyond the three-dimensional version of the polyhedral formula given by Lakatos; it is dimension-free, in the sense that it gives a formula in which the dimension of the polyhedron is a parameter. The classical Euler relation $V-E+F=2$ is corresponds to the case where the dimension of the polyhedron is 3 .

The main theorem, expressed in the language of the present article, is

$$
\text { Sum alternating - characteristic }- \text { sequence }(p)=0 \text {, }
$$

where $p$ is a polyhedron. The alternating characteristic sequence of a polyhedron is the sequence

$$
-N(-1),+N(0),-N(1), \ldots,(-1)^{\operatorname{dim}(p)} * N(\operatorname{dim}(p)),
$$

where $N(k)$ is the number of polytopes of $p$ of dimension $k$. The special case of $\operatorname{dim}(p)=3$ yields Euler's classical relation. $(N(-1)$ and $N(3)$ will turn out to be equal, by definition, to 1 .)

Two other special cases are proved: the first says that a one-dimensional "polyhedron" that is a homology sphere consists of just two vertices (and thus consists of just a single edge); the second special case asserts that a two-dimensional polyhedron that is a homology sphere (a polygon) has as many vertices as edges.

A treatment of the more general version of Euler's relation can be found in [12] and [6]. The former contains a proof of Steinitz's theorem, which shows 
that the abstract polyhedra treated in Poincaré's proof, which might not appear to be about polyhedra in the usual sense of the word, are in fact embeddable in $\mathbf{R}^{3}$ under certain conditions. It would be valuable to formalize a proof of Steinitz's theorem and relate it to the development contained here.

MML identifier: POLYFORM, version: $\underline{7.8 .05 \quad 4.89 .993}$

The terminology and notation used here are introduced in the following articles: $[9],[27],[28],[7],[8],[21],[10],[4],[22],[3],[5],[14],[19],[26],[23],[13],[25]$, $[24],[16],[20],[29],[1]$, and [2].

\section{Set-theoretical Preliminaries}

The following propositions are true:

(1) For all sets $X, c, d$ such that there exist sets $a, b$ such that $a \neq b$ and $X=\{a, b\}$ and $c, d \in X$ and $c \neq d$ holds $X=\{c, d\}$.

(2) For every function $f$ such that $f$ is one-to-one holds $\overline{\overline{\operatorname{dom} f}}=\overline{\overline{\operatorname{nng} f}}$.

\section{Arithmetical Preliminaries}

In the sequel $n$ denotes a natural number and $k$ denotes an integer.

Next we state the proposition

(3) If $1 \leq k$, then $k$ is a natural number.

Let $a$ be an integer and let $b$ be a natural number. Then $a \cdot b$ is an element of $\mathbb{Z}$.

One can prove the following propositions:

(4) 1 is odd.

(5) 2 is even.

(6) 3 is odd.

(7) 4 is even.

(8) If $n$ is even, then $(-1)^{n}=1$

(9) If $n$ is odd, then $(-1)^{n}=-1$.

(10) $(-1)^{n}$ is an integer.

Let $a$ be an integer and let $n$ be a natural number. Then $a^{n}$ is an element of $\mathbb{Z}$.

We now state four propositions:

(11) For all finite sequences $p, q, r$ holds $\operatorname{len}\left(p^{\frown} q\right) \leq \operatorname{len}\left(p^{\frown}\left(q^{\frown} r\right)\right)$. 
(12) $1<n+2$.

(13) $(-1)^{2}=1$.

(14) For every natural number $n$ holds $(-1)^{n}=(-1)^{n+2}$.

\section{Preliminaries on Finite Sequences}

Let $f$ be a finite sequence of elements of $\mathbb{Z}$ and let $k$ be a natural number. Observe that $f_{k}$ is integer.

The following propositions are true:

(15) Let $a, b, s$ be finite sequences of elements of $\mathbb{Z}$. Suppose that

(i) $\quad$ len $s>0$,

(ii) $\operatorname{len} a=$ len $s$,

(iii) $\quad$ len $s=\operatorname{len} b$,

(iv) for every natural number $n$ such that $1 \leq n \leq$ len $s$ holds $s_{n}=a_{n}+b_{n}$, and

(v) for every natural number $k$ such that $1 \leq k<$ len $s$ holds $b_{k}=-a_{k+1}$. Then $\sum s=a_{1}+b_{\text {len } s}$.

(16) For all finite sequences $p, q, r$ holds $\operatorname{len}\left(p^{\frown} q^{\frown} r\right)=\operatorname{len} p+\operatorname{len} q+\operatorname{len} r$.

(17) For every set $x$ and for all finite sequences $p, q$ holds $\left(\langle x\rangle^{\frown} p^{\frown} q\right)_{1}=x$.

(18) For every set $x$ and for all finite sequences $p, q$ holds $\left(p^{\frown} q^{\frown}\right.$ $\langle x\rangle)_{\operatorname{len} p+\operatorname{len} q+1}=x$.

(19) For all finite sequences $p, q, r$ and for every natural number $k$ such that len $p<k \leq \operatorname{len}\left(p^{\frown} q\right)$ holds $\left(p^{\frown} q^{\frown} r\right)_{k}=q_{k-\operatorname{len} p}$.

Let $a$ be an integer. Then $\langle a\rangle$ is a finite sequence of elements of $\mathbb{Z}$.

Let $a, b$ be integers. Then $\langle a, b\rangle$ is a finite sequence of elements of $\mathbb{Z}$.

Let $a, b, c$ be integers. Then $\langle a, b, c\rangle$ is a finite sequence of elements of $\mathbb{Z}$.

Let $p, q$ be finite sequences of elements of $\mathbb{Z}$. Then $p^{\frown} q$ is a finite sequence of elements of $\mathbb{Z}$.

We now state four propositions:

(20) For all finite sequences $p, q$ of elements of $\mathbb{Z}$ holds $\sum p^{\frown} q=\left(\sum p\right)+\sum q$.

(21) For every integer $k$ and for every finite sequence $p$ of elements of $\mathbb{Z}$ holds $\sum\langle k\rangle \frown p=k+\sum p$.

(22) For all finite sequences $p, q, r$ of elements of $\mathbb{Z}$ holds $\sum p^{\frown} q^{\frown} r=$ $\left(\sum p\right)+\sum q+\sum r$.

(23) For every element $a$ of $\mathbf{Z}_{2}$ holds $\sum\langle a\rangle=a$. 


\section{Polyhedra And Incidence Matrices}

Let $X, Y$ be sets. An incidence matrix of $X$ and $Y$ is an element of $\left\{0 \mathbf{Z}_{2}, 1_{\mathbf{Z}_{2}}\right\}^{X \times Y}$.

We now state the proposition

(24) For all sets $X, Y$ holds $X \times Y \longmapsto 1_{\mathbf{z}_{2}}$ is an incidence matrix of $X$ and $Y$.

Polyhedron is defined by the condition (Def. 1).

(Def. 1) There exists a finite sequence-yielding finite sequence $F$ and there exists a function yielding finite sequence $I$ such that

(i) $\quad$ len $I=\operatorname{len} F-1$,

(ii) for every natural number $n$ such that $1 \leq n<\operatorname{len} F$ holds $I(n)$ is an incidence matrix of $\operatorname{rng} F(n)$ and $\operatorname{rng} F(n+1)$,

(iii) for every natural number $n$ such that $1 \leq n \leq \operatorname{len} F$ holds $F(n)$ is non empty and $F(n)$ is one-to-one, and

(iv) it $=\langle F, I\rangle$.

In the sequel $p$ denotes a polyhedron, $k$ denotes an integer, and $n$ denotes a natural number.

Let us consider $p$. Then $p_{\mathbf{1}}$ is a finite sequence-yielding finite sequence. Then $p_{\mathbf{2}}$ is a function yielding finite sequence.

Let $p$ be a polyhedron. The functor $\operatorname{dim}(p)$ yielding an element of $\mathbb{N}$ is defined by:

(Def. 2) $\operatorname{dim}(p)=\operatorname{len}\left(p_{1}\right)$.

Let $p$ be a polyhedron and let $k$ be an integer. The functor $P_{k, p}$ yielding a finite set is defined by the conditions (Def. 3).

(Def. 3)(i) If $k<-1$, then $P_{k, p}=\emptyset$,

(ii) if $k=-1$, then $P_{k, p}=\{\emptyset\}$,

(iii) if $-1<k<\operatorname{dim}(p)$, then $P_{k, p}=\operatorname{rng} p_{\mathbf{1}}(k+1)$,

(iv) if $k=\operatorname{dim}(p)$, then $P_{k, p}=\{p\}$, and

(v) if $k>\operatorname{dim}(p)$, then $P_{k, p}=\emptyset$.

One can prove the following two propositions:

(25) If $-1<k<\operatorname{dim}(p)$, then $k+1$ is a natural number and $1 \leq k+1 \leq$ $\operatorname{dim}(p)$.

(26) $P_{k, p}$ is non empty iff $-1 \leq k \leq \operatorname{dim}(p)$.

Let $p$ be a polyhedron and let $k$ be an integer. Let us assume that $-1 \leq k \leq$ $\operatorname{dim}(p) . k$-polytope of $p$ is defined by:

(Def. 4) $\quad$ It $\in P_{k, p}$.

Next we state the proposition

(27) If $k<\operatorname{dim}(p)$, then $k-1<\operatorname{dim}(p)$. 
Let $p$ be a polyhedron and let $k$ be an integer. The functor $\eta_{p, k}$ yielding an incidence matrix of $P_{k-1, p}$ and $P_{k, p}$ is defined by the conditions (Def. 5).

(Def. 5)(i) If $k<0$, then $\eta_{p, k}=\emptyset$,

(ii) if $k=0$, then $\eta_{p, k}=\{\emptyset\} \times P_{0, p} \longmapsto 1_{\mathbf{Z}_{2}}$,

(iii) if $0<k<\operatorname{dim}(p)$, then $\eta_{p, k}=p_{\mathbf{2}}(k)$,

(iv) $\quad$ if $k=\operatorname{dim}(p)$, then $\eta_{p, k}=P_{\operatorname{dim}(p)-1, p} \times\{p\} \longmapsto 1_{\mathbf{z}_{2}}$, and

(v) if $k>\operatorname{dim}(p)$, then $\eta_{p, k}=\emptyset$.

Let $p$ be a polyhedron and let $k$ be an integer. The functor $S_{k, p}$ yielding a finite sequence is defined by the conditions (Def. 6).

(Def. 6)(i) If $k<-1$, then $S_{k, p}=\varepsilon_{\emptyset}$,

(ii) if $k=-1$, then $S_{k, p}=\langle\emptyset\rangle$,

(iii) if $-1<k<\operatorname{dim}(p)$, then $S_{k, p}=p_{\mathbf{1}}(k+1)$,

(iv) if $k=\operatorname{dim}(p)$, then $S_{k, p}=\langle p\rangle$, and

(v) if $k>\operatorname{dim}(p)$, then $S_{k, p}=\varepsilon_{\emptyset}$.

Let $p$ be a polyhedron and let $k$ be an integer. The functor $N_{p, k}$ yielding an element of $\mathbb{N}$ is defined as follows:

(Def. 7) $\quad N_{p, k}=\overline{\overline{P_{k, p}}}$.

Let $p$ be a polyhedron. The functor $V_{p}$ yields an element of $\mathbb{N}$ and is defined by:

(Def. 8) $V_{p}=N_{p, 0}$.

The functor $E_{p}$ yields an element of $\mathbb{N}$ and is defined by:

(Def. 9) $\quad E_{p}=N_{p, 1}$.

The functor $F_{p}$ yielding an element of $\mathbb{N}$ is defined by:

(Def. 10) $\quad F_{p}=N_{p, 2}$.

Next we state several propositions:

(28) $\operatorname{dom}\left(S_{k, p}\right)=\operatorname{Seg}\left(N_{p, k}\right)$.

(29) $\operatorname{len}\left(S_{k, p}\right)=N_{p, k}$.

(30) $\operatorname{rng}\left(S_{k, p}\right)=P_{k, p}$.

(31) $\quad N_{p,-1}=1$.

(32) $\quad N_{p, \operatorname{dim}(p)}=1$

Let $p$ be a polyhedron, let $k$ be an integer, and let $n$ be a natural number. Let us assume that $1 \leq n \leq N_{p, k}$ and $-1 \leq k \leq \operatorname{dim}(p)$. The functor $P_{p, k}^{n}$ yielding an element of $P_{k, p}$ is defined by:

(Def. 11) $P_{p, k}^{n}=S_{k, p}(n)$.

We now state three propositions:

(33) Suppose $-1 \leq k \leq \operatorname{dim}(p)$. Let $x$ be a $k$-polytope of $p$. Then there exists a natural number $n$ such that $x=P_{p, k}^{n}$ and $1 \leq n \leq N_{p, k}$.

(34) $S_{k, p}$ is one-to-one. 
(35) Suppose $-1 \leq k \leq \operatorname{dim}(p)$. Let $m, n$ be natural numbers. If $1 \leq n \leq N_{p, k}$ and $1 \leq m \leq N_{p, k}$ and $P_{p, k}^{n}=P_{p, k}^{m}$, then $m=n$.

Let $p$ be a polyhedron, let $k$ be an integer, let $x$ be a $(k-1)$-polytope of $p$, and let $y$ be a $k$-polytope of $p$. Let us assume that $0 \leq k \leq \operatorname{dim}(p)$. The functor $x(y)$ yields an element of $\mathbf{Z}_{2}$ and is defined by:

(Def. 12) $\quad x(y)=\eta_{p, k}(x, y)$.

\section{The Chain Spaces and their Subspaces. Boundary of a $k$-Chain}

Let $p$ be a polyhedron and let $k$ be an integer. The functor $C_{k, p}$ yielding a finite dimensional vector space over $\mathbf{Z}_{2}$ is defined by:

(Def. 13) $\quad C_{k, p}=B_{P_{k, p}}$.

We now state two propositions:

(36) For every $k$-polytope $x$ of $p$ holds $0_{C_{k, p}}{ }^{\circledR} x=0 \mathbf{Z}_{2}$.

(37) $N_{p, k}=\operatorname{dim}\left(C_{k, p}\right)$.

Let $p$ be a polyhedron and let $k$ be an integer. The functor $k$-chains $p$ yielding a non empty finite set is defined by:

(Def. 14) $k$-chains $p=2^{P_{k, p}}$.

Let $p$ be a polyhedron, let $k$ be an integer, let $x$ be a $(k-1)$-polytope of $p$, and let $v$ be an element of $C_{k, p}$. The functor $v(x)$ yielding a finite sequence of elements of $\mathbf{Z}_{2}$ is defined by the conditions (Def. 15).

(Def. 15)(i) If $P_{k-1, p}$ is empty, then $v(x)=\varepsilon_{\emptyset}$, and

(ii) if $P_{k-1, p}$ is non empty, then $\operatorname{len}(v(x))=N_{p, k}$ and for every natural number $n$ such that $1 \leq n \leq N_{p, k}$ holds $v(x)(n)=\left(v^{@} P_{p, k}^{n}\right) \cdot x\left(P_{p, k}^{n}\right)$.

We now state several propositions:

(38) For all elements $c, d$ of $C_{k, p}$ and for every $k$-polytope $x$ of $p$ holds $(c+$ $d)^{@} x=c^{@} x+d^{@} x$.

(39) For all elements $c, d$ of $C_{k, p}$ and for every $(k-1)$-polytope $x$ of $p$ holds $(c+d)(x)=c(x)+d(x)$.

(40) For all elements $c, d$ of $C_{k, p}$ and for every $(k-1)$-polytope $x$ of $p$ holds $\sum(c(x)+d(x))=\left(\sum c(x)\right)+\sum d(x)$.

(41) For all elements $c, d$ of $C_{k, p}$ and for every $(k-1)$-polytope $x$ of $p$ holds $\sum(c+d)(x)=\left(\sum c(x)\right)+\sum d(x)$.

(42) For every element $c$ of $C_{k, p}$ and for every element $a$ of $\mathbf{Z}_{2}$ and for every $k$-polytope $x$ of $p$ holds $(a \cdot c)^{@} x=a \cdot\left(c^{@} x\right)$.

(43) For every element $c$ of $C_{k, p}$ and for every element $a$ of $\mathbf{Z}_{2}$ and for every $k$-polytope $x$ of $p$ holds $(a \cdot c)(x)=a \cdot c(x)$.

(44) For all elements $c, d$ of $C_{k, p}$ holds $c=d$ iff for every $k$-polytope $x$ of $p$ holds $c^{@} x=d^{@} x$. 
(45) For all elements $c, d$ of $C_{k, p}$ holds $c=d$ iff for every $k$-polytope $x$ of $p$ holds $x \in c$ iff $x \in d$.

The scheme ChainEx deals with a polyhedron $\mathcal{A}$, an integer $\mathcal{B}$, and a unary predicate $\mathcal{P}$, and states that:

There exists a subset $c$ of $P_{\mathcal{B}, \mathcal{A}}$ such that for every $\mathcal{B}$-polytope $x$ of $\mathcal{A}$ holds $x \in c$ iff $\mathcal{P}[x]$ and $x \in P_{\mathcal{B}, \mathcal{A}}$

for all values of the parameters.

Let $p$ be a polyhedron, let $k$ be an integer, and let $v$ be an element of $C_{k, p}$. The functor $\partial v$ yields an element of $C_{k-1, p}$ and is defined by the conditions (Def. 16).

(Def. 16)(i) If $P_{k-1, p}$ is empty, then $\partial v=0_{C_{k-1, p}}$, and

(ii) if $P_{k-1, p}$ is non empty, then for every $(k-1)$-polytope $x$ of $p$ holds $x \in \partial v$ iff $\sum v(x)=1_{\mathbf{Z}_{2}}$.

One can prove the following proposition

(46) For every element $c$ of $C_{k, p}$ and for every $(k-1)$-polytope $x$ of $p$ holds $\partial c^{@} x=\sum c(x)$.

Let $p$ be a polyhedron and let $k$ be an integer. The functor $\partial_{k} p$ yields a function from $C_{k, p}$ into $C_{k-1, p}$ and is defined by:

(Def. 17) For every element $c$ of $C_{k, p}$ holds $\partial_{k} p(c)=\partial c$.

One can prove the following propositions:

(47) For all elements $c, d$ of $C_{k, p}$ holds $\partial(c+d)=\partial c+\partial d$.

(48) For every element $a$ of $\mathbf{Z}_{2}$ and for every element $c$ of $C_{k, p}$ holds $\partial(a \cdot c)=$ $a \cdot \partial c$.

(49) $\partial_{k} p$ is a linear transformation from $C_{k, p}$ to $C_{k-1, p}$.

Let $p$ be a polyhedron and let $k$ be an integer. Then $\partial_{k} p$ is a linear transformation from $C_{k, p}$ to $C_{k-1, p}$.

Let $p$ be a polyhedron and let $k$ be an integer. The functor $Z_{k, p}$ yielding a subspace of $C_{k, p}$ is defined as follows:

(Def. 18) $\quad Z_{k, p}=\operatorname{ker} \partial_{k} p$.

Let $p$ be a polyhedron and let $k$ be an integer. The functor $\left|Z_{k, p}\right|$ yields a non empty subset of $k$-chains $p$ and is defined by:

(Def. 19) $\left|Z_{k, p}\right|=\Omega_{Z_{k, p}}$.

Let $p$ be a polyhedron and let $k$ be an integer. The functor $B_{k, p}$ yields a subspace of $C_{k, p}$ and is defined as follows:

(Def. 20) $\quad B_{k, p}=\operatorname{im}\left(\partial_{k+1} p\right)$.

Let $p$ be a polyhedron and let $k$ be an integer. The functor $\left|B_{k, p}\right|$ yielding a non empty subset of $k$-chains $p$ is defined by:

(Def. 21) $\left|B_{k, p}\right|=\Omega_{B_{k, p}}$. 
Let $p$ be a polyhedron and let $k$ be an integer. The functor $\mathrm{BZ}_{k, p}$ yields a subspace of $C_{k, p}$ and is defined as follows:

(Def. 22) $\quad \mathrm{BZ}_{k, p}=B_{k, p} \cap Z_{k, p}$.

Let $p$ be a polyhedron and let $k$ be an integer.

The functor $k$-bounding-circuits $p$ yields a non empty subset of $k$-chains $p$ and is defined as follows:

(Def. 23) $\quad k$-bounding-circuits $p=\Omega_{\mathrm{BZ}}$.

The following proposition is true

(50) $\operatorname{dim}\left(C_{k, p}\right)=\operatorname{rank}\left(\partial_{k} p\right)+\operatorname{nullity}\left(\partial_{k} p\right)$.

\section{Simply Connected And Eulerian Polyhedra}

Let $p$ be a polyhedron. We say that $p$ is being a homology sphere if and only if:

(Def. 24) For every integer $k$ holds $\left|Z_{k, p}\right|=\left|B_{k, p}\right|$.

The following proposition is true

(51) $\quad p$ is being a homology sphere iff for every integer $n$ holds $Z_{n, p}=B_{n, p}$.

Let $p$ be a polyhedron. The functor $\widehat{p}$ yielding a finite sequence of elements of $\mathbb{Z}$ is defined as follows:

(Def. 25) len $\widehat{p}=\operatorname{dim}(p)+2$ and for every natural number $k$ such that $1 \leq k \leq$ $\operatorname{dim}(p)+2$ holds $\widehat{p}(k)=(-1)^{k} \cdot N_{p, k-2}$.

Let $p$ be a polyhedron. The functor $\bar{p}$ yields a finite sequence of elements of $\mathbb{Z}$ and is defined by:

(Def. 26) $\operatorname{len} \bar{p}=\operatorname{dim}(p)$ and for every natural number $k$ such that $1 \leq k \leq \operatorname{dim}(p)$ holds $\bar{p}(k)=(-1)^{k+1} \cdot N_{p, k-1}$.

Let $p$ be a polyhedron. The functor $\bar{p}$ yielding a finite sequence of elements of $\mathbb{Z}$ is defined as follows:

(Def. 27) $\operatorname{len} \bar{p}=\operatorname{dim}(p)+1$ and for every natural number $k$ such that $1 \leq k \leq$ $\operatorname{dim}(p)+1$ holds $\bar{p}(k)=(-1)^{k+1} \cdot N_{p, k-1}$.

One can prove the following proposition

(52) If $1 \leq n \leq$ len $\bar{p}$, then $\bar{p}(n)=(-1)^{n+1} \cdot \operatorname{dim}\left(B_{n-2, p}\right)+(-1)^{n+1}$. $\operatorname{dim}\left(Z_{n-1, p}\right)$.

Let $p$ be a polyhedron. We say that $p$ is Eulerian if and only if:

(Def. 28) $\sum \bar{p}=1+(-1)^{\operatorname{dim}(p)+1}$.

One can prove the following proposition

(53) $\bar{p}=\bar{p}^{\frown}\left\langle(-1)^{\operatorname{dim}(p)}\right\rangle$.

Let $p$ be a polyhedron. Let us observe that $p$ is Eulerian if and only if:

(Def. 29) $\sum \bar{p}=1$. 
One can prove the following proposition

(54) $\hat{p}=\langle-1\rangle^{\frown} \bar{p}$.

Let $p$ be a polyhedron. Let us observe that $p$ is Eulerian if and only if:

(Def. 30) $\sum \widehat{p}=0$.

\section{The Extremal Chain Spaces}

The following propositions are true:

(55) $P_{0, p}$ is non empty.

(56) $\overline{\overline{\Omega_{C_{-1, p}}}}=2$.

(57) $\Omega_{C_{-1, p}}=\{\emptyset,\{\emptyset\}\}$.

(58) For every $k$-polytope $x$ of $p$ and for every $(k-1)$-polytope $e$ of $p$ such that $k=0$ and $e=\emptyset$ holds $e(x)=1_{\mathbf{z}_{2}}$.

(59) Let $k$ be an integer, $x$ be a $k$-polytope of $p, v$ be an element of $C_{k, p}, e$ be a $(k-1)$-polytope of $p$, and $n$ be a natural number. If $k=0$ and $v=\{x\}$ and $e=\emptyset$ and $x=P_{p, k}^{n}$ and $1 \leq n \leq N_{p, k}$, then $v(e)(n)=1_{\mathbf{z}_{2}}$.

(60) Let $k$ be an integer, $x$ be a $k$-polytope of $p, e$ be a $(k-1)$-polytope of $p$, $v$ be an element of $C_{k, p}$, and $m, n$ be natural numbers. Suppose $k=0$ and $v=\{x\}$ and $x=P_{p, k}^{n}$ and $1 \leq m \leq N_{p, k}$ and $1 \leq n \leq N_{p, k}$ and $m \neq n$. Then $v(e)(m)=0 \mathbf{Z}_{2}$.

(61) Let $k$ be an integer, $x$ be a $k$-polytope of $p, v$ be an element of $C_{k, p}$, and $e$ be a $(k-1)$-polytope of $p$. If $k=0$ and $v=\{x\}$ and $e=\emptyset$, then $\sum v(e)=1_{\mathbf{Z}_{2}}$.

(62) For every 0-polytope $x$ of $p$ holds $\partial_{0} p(\{x\})=\{\emptyset\}$.

(63) $\operatorname{dim}\left(B_{(-1), p}\right)=1$.

(64) $\overline{\overline{\Omega_{C_{\operatorname{dim}(p), p}}}}=2$.

(65) $\{p\}$ is an element of $C_{\operatorname{dim}(p), p}$.

(66) $\{p\} \in \Omega_{C_{\operatorname{dim}(p), p}}$.

(67) $\quad P_{\operatorname{dim}(p)-1, p}$ is non empty.

Let $p$ be a polyhedron. Note that $P_{\operatorname{dim}(p)-1, p}$ is non empty.

The following propositions are true:

(68) $\Omega_{C_{\operatorname{dim}(p), p}}=\left\{0_{C_{\operatorname{dim}(p), p}},\{p\}\right\}$.

(69) For every element $x$ of $C_{\operatorname{dim}(p), p}$ holds $x=0_{C_{\operatorname{dim}(p), p}}$ or $x=\{p\}$.

(70) For all elements $x, y$ of $C_{\operatorname{dim}(p), p}$ such that $x \neq y$ holds $x=0_{C_{\operatorname{dim}(p), p}}$ or $y=0_{C_{\operatorname{dim}(p), p}}$.

(71) $S_{\operatorname{dim}(p), p}=\langle p\rangle$.

(72) $P_{p, \operatorname{dim}(p)}^{1}=p$. 
(73) For every element $c$ of $C_{\operatorname{dim}(p), p}$ and for every $\operatorname{dim}(p)$-polytope $x$ of $p$ such that $c=\{p\}$ holds $c{ }^{\circledR} x=1 \mathbf{z}_{2}$.

(74) For every $(\operatorname{dim}(p)-1)$-polytope $x$ of $p$ and for every $\operatorname{dim}(p)$-polytope $c$ of $p$ such that $c=p$ holds $x(c)=1 \mathbf{z}_{2}$.

(75) For every $(\operatorname{dim}(p)-1)$-polytope $x$ of $p$ and for every element $c$ of $C_{\operatorname{dim}(p), p}$ such that $c=\{p\}$ holds $c(x)=\left\langle 1 \mathbf{z}_{2}\right\rangle$.

(76) For every $(\operatorname{dim}(p)-1)$-polytope $x$ of $p$ and for every element $c$ of $C_{\operatorname{dim}(p), p}$ such that $c=\{p\}$ holds $\sum c(x)=1 \mathbf{z}_{2}$.

(77) $\partial_{\operatorname{dim}(p)} p(\{p\})=P_{\operatorname{dim}(p)-1, p}$

(78) $\partial_{\operatorname{dim}(p)} p$ is one-to-one.

(79) $\operatorname{dim}\left(B_{\operatorname{dim}(p)-1, p}\right)=1$.

(80) If $p$ is being a homology sphere, then $\operatorname{dim}\left(Z_{\operatorname{dim}(p)-1, p}\right)=1$.

(81) If $1<n<\operatorname{dim}(p)+2$, then $\widehat{p}(n)=\bar{p}(n-1)$.

(82) $\widehat{p}=\langle-1\rangle^{\frown} \bar{p}^{\frown}\left\langle(-1)^{\operatorname{dim}(p)}\right\rangle$.

\section{A Generalized Euler Relation and its 1-, 2-, And 3-Dimensional Special Cases}

One can prove the following propositions:

(83) If $\operatorname{dim}(p)$ is odd, then $\sum \widehat{p}=\left(\sum \bar{p}\right)-2$.

(84) If $\operatorname{dim}(p)$ is even, then $\sum \widehat{p}=\sum \bar{p}$.

(85) If $\operatorname{dim}(p)=1$, then $\sum \bar{p}=N_{p, 0}$.

(86) If $\operatorname{dim}(p)=2$, then $\sum \bar{p}=N_{p, 0}-N_{p, 1}$.

(87) If $\operatorname{dim}(p)=3$, then $\sum \bar{p}=\left(N_{p, 0}-N_{p, 1}\right)+N_{p, 2}$.

(88) If $\operatorname{dim}(p)=0$, then $p$ is Eulerian.

(89) If $p$ is being a homology sphere, then $p$ is Eulerian.

(90) If $p$ is being a homology sphere and $\operatorname{dim}(p)=1$, then $V_{p}=2$.

(91) If $p$ is being a homology sphere and $\operatorname{dim}(p)=2$, then $V_{p}=E_{p}$.

(92) If $p$ is being a homology sphere and $\operatorname{dim}(p)=3$, then $\left(V_{p}-E_{p}\right)+F_{p}=2$.

\section{REFERENCES}

[1] Jesse Alama. The rank+nullity theorem. Formalized Mathematics, 15(3):137-142, 2007.

[2] Jesse Alama. The vector space of subsets of a set based on symmetric difference. Formalized Mathematics, 16(1):1-5, 2008.

[3] Grzegorz Bancerek. Cardinal numbers. Formalized Mathematics, 1(2):377-382, 1990.

[4] Grzegorz Bancerek. The fundamental properties of natural numbers. Formalized Mathematics, 1(1):41-46, 1990.

[5] Grzegorz Bancerek and Krzysztof Hryniewiecki. Segments of natural numbers and finite sequences. Formalized Mathematics, 1(1):107-114, 1990.

[6] Arne Brøndsted. An Introduction to Convex Polytopes. Graduate Texts in Mathematics. Springer, 1983. 
[7] Czesław Byliński. Functions and their basic properties. Formalized Mathematics, 1(1):5565, 1990.

[8] Czesław Byliński. Functions from a set to a set. Formalized Mathematics, 1(1):153-164, 1990.

[9] Czesław Byliński. Some basic properties of sets. Formalized Mathematics, 1(1):47-53, 1990.

[10] Agata Darmochwał. Finite sets. Formalized Mathematics, 1(1):165-167, 1990.

[11] Leonhard Euler. Elementa doctrinae solidorum. Novi Commentarii Academiae Scientarum Petropolitanae, 4:109-140, 1758.

[12] Branko Grünbaum. Convex Polytopes. Number 221 in Graduate Texts in Mathematics. Springer, 2nd edition, 2003.

[13] Eugeniusz Kusak, Wojciech Leończuk, and Michał Muzalewski. Abelian groups, fields and vector spaces. Formalized Mathematics, 1(2):335-342, 1990.

[14] Rafał Kwiatek. Factorial and Newton coefficients. Formalized Mathematics, 1(5):887-890, 1990.

[15] Imre Lakatos. Proofs and Refutations: The Logic of Mathematical Discovery. Cambridge University Press, 1976. Edited by John Worrall and Elie Zahar.

[16] Michał Muzalewski. Rings and modules - part II. Formalized Mathematics, 2(4):579-585, 1991.

[17] Henri Poincaré. Sur la généralisation d'un théorème d'Euler relatif aux polyèdres. Comptes Rendus de Séances de l'Academie des Sciences, 117:144, 1893.

[18] Henri Poincaré. Complément à l'analysis situs. Rendiconti del Circolo Matematico di Palermo, 13:285-343, 1899.

[19] Piotr Rudnicki and Andrzej Trybulec. Abian's fixed point theorem. Formalized Mathematics, 6(3):335-338, 1997.

[20] Dariusz Surowik. Cyclic groups and some of their properties - part I. Formalized Mathematics, 2(5):623-627, 1991.

[21] Andrzej Trybulec. Binary operations applied to functions. Formalized Mathematics, 1(2):329-334, 1990.

[22] Michał J. Trybulec. Integers. Formalized Mathematics, 1(3):501-505, 1990.

[23] Wojciech A. Trybulec. Groups. Formalized Mathematics, 1(5):821-827, 1990.

[24] Wojciech A. Trybulec. Linear combinations in vector space. Formalized Mathematics, $1(5): 877-882,1990$

[25] Wojciech A. Trybulec. Subspaces and cosets of subspaces in vector space. Formalized Mathematics, 1(5):865-870, 1990.

[26] Wojciech A. Trybulec. Vectors in real linear space. Formalized Mathematics, 1(2):291-296, 1990.

[27] Zinaida Trybulec. Properties of subsets. Formalized Mathematics, 1(1):67-71, 1990.

[28] Edmund Woronowicz. Relations and their basic properties. Formalized Mathematics, 1(1):73-83, 1990.

[29] Mariusz Żynel. The Steinitz theorem and the dimension of a vector space. Formalized Mathematics, 5(3):423-428, 1996.

Received October 9, 2007 